\title{
When do primes go bad? A corpus of orthographically related primes that inhibit fragment completion
}

\author{
Olga Rass ANd P. Andrew Leynes \\ College of New Jersey, Ewing, New Jersey
}

\begin{abstract}
The memory block effect (MBE) occurs when orthographically similar words inhibit retrieval. Previous studies have published 55 different stimuli that produce the MBE in word fragment completion. This small number of stimuli constrains experimental designs, presents serious obstacles for using neuroimaging to elucidate neural substrates of blocking, and raises concern that the MBE is limited to a particular group of words. A pool of 315 stimulus words was tested in a traditional MBE paradigm, and the results demonstrated that the MBE generalizes to other stimuli. This study also expands the number of stimuli that produce the MBE because 185 new stimuli produced blocking effects. As a result, the current list of $240 \mathrm{MBE}$ stimuli can be used for word fragment research including cognitive neuroscience investigations of retrieval inhibition. A table of MBE stimuli is available in an archived appendix that can be downloaded from www.psychonomic.org/archive.
\end{abstract}

Retrieval failures are part of our everyday cognition. For example, recalling the capital of Australia can often create momentary confusion when one quickly realizes the famous city of Sydney is not the capital. Cues can aid retrieval in many cases, such as when $C A N O P Y$ primes the retrieval of Australia's capital because it has the same first three letters and the same number of syllables as the correct answer. However, recent memory research has demonstrated that partial cuing with orthographically similar words inhibits retrieval of target words (Logan \& Balota, 2003; Lustig \& Hasher, 2001). For example, the cue $C R A N B E R R Y$ might slow or block successful retrieval because it is orthographically similar to the name of Australia's capital (Canberra).

The memory block effect (MBE) refers to cases in which an orthographically similar word impedes the retrieval of a known word. This effect has been studied in the laboratory using a word fragment completion task (Landau \& Leynes, 2006; Smith \& Tindell, 1997). During the first part of this task, participants are exposed to words that complete test fragments (positive primes) and other words that are orthographically similar to the solutions but cannot complete the fragment (blocking primes). Memory is then tested using an incidental memory test that involves completing word fragments. Control conditions consist of studying unrelated words (Kinoshita \& Towgood, 2001; Smith \& Tindell, 1997), studying unrelated primes or no prime exposure (Logan \& Balota, 2003; Lustig \& Hasher, 2001 ), or only no prime exposure (Landau \& Leynes, 2006) in order to measure base rates of fragment completion. Typical results reveal that studying the blocking prime (e.g., $B A L L O O N$ ) decreases the probability that the corresponding word fragment (BAL_ON_) will be completed (Kinoshita \& Towgood, 2001; Landau \& Leynes, 2006; Logan \& Balota, 2003; Lustig \& Hasher, 2001; Smith \& Tindell, 1997), whereas seeing the positive prime $(B A L C O N Y)$ will increase the probability of completing the fragment relative to control conditions (Kinoshita \& Towgood, 2001; Smith \& Tindell, 1997). Such results support the idea that exposure to blocking primes disrupts the ability to retrieve words that complete the fragments and represents a case of retrieval inhibition.

Unfortunately, a clear theoretical explanation of the MBE mechanism has not emerged. Exploring its limits has determined that the effect is robust. The magnitude of the MBE is unaffected by conscious versus subthreshold presentation of study primes, different encoding tasks, increased time allotted to solve the fragment, and instructions to ignore blocking words (Landau \& Leynes, 2006; Logan \& Balota, 2003; Smith \& Tindell, 1997). However, Smith and Tindell reported significant blocking when the study and test modality was visual, but did not observe blocking when primes were heard at study and the fragments were presented visually. Based on results across several studies, Smith and Tindell suggested that involuntary retrieval of negative primes resulted in unconscious blocks of memory, thereby suggesting that the MBE results from the influence of implicit memory. Alternatively, Kinoshita and Towgood (2001) claimed that the MBE results from spontaneous recognition (i.e., explicit memory) of the blocking primes when they observed a decreased MBE with divided attention at encoding. Their claim has been challenged recently by evidence that

P.A. Leynes, leynes@tcnj.edu 
the MBE magnitude is unaffected by manipulations that enhance recognition (Landau \& Leynes, 2006; see also Lustig $\&$ Hasher, 2001). As a result, the mechanism that produces the MBE phenomenon remains an unresolved issue.

Studying the MBE using word fragment completion has several advantages. First, it is similar to other retrieval inhibition phenomena, including directed forgetting, retrievalinduced forgetting, negative priming, feeling-of-knowing (FOK), and tip-of-the-tongue (TOT) effects (Landau \& Leynes, 2006; Logan \& Balota, 2003; Smith \& Tindell, 1997). Therefore, defining the MBE mechanism can potentially provide a better understanding of retrieval inhibition models and everyday memory failures, such as lexical retrieval difficulties that are common occurrences in older adults (Logan \& Balota, 2003). Second, the simple design yields objective measures of memory (e.g., proportion of errors, completions, etc.), rather than relying on subjective assessments of memory (e.g., FOK, TOT) that often have complicated instructions. Finally, understanding the MBE may result in applications that help patients with neurological disorders that include memory dysfunctions, such as repression, obsessive-compulsive disorder, and Alzheimer's disease (Logan \& Balota, 2003; Smith \& Tindell, 1997).

The lack of a substantial pool of MBE stimuli continues to be a limitation for using a word fragment paradigm. Table 1 displays the total number of unique stimuli (55) yielded by past studies (Kinoshita \& Towgood, 2001; Logan \& Balota, 2003; Smith \& Tindell, 1997). ${ }^{1}$ In fact, the conclusions from these studies were based on only a few trials in each experimental condition (range, 4-16 items/condition). A larger pool of stimuli is desirable for a number of reasons. First, testing a larger number of stimuli has the potential to demonstrate that the MBE is not limited to a special class of stimuli. Second, a larger stimulus pool would increase power and enable matching various conditions based on a number of variables, such as priming/blocking magnitude, base rate of fragment completion, word frequency, etc. Third, a larger pool would allow measurement of neural activity using neuroimaging technology, such as functional magnetic resonance imaging (fMRI) and event-related potentials (ERPs), because this technology requires multiple stimulus presentations in order to average out background (random) brain activity. For example, ERP studies require a minimum of 10 to 15 trials per condition in most applications in order to obtain acceptable signal-to-noise ratios for ERP averages (Pincton et al., 2000), and stimuli cannot be repeated in ERP memory studies because mere repetition influences ERP activity (see Rugg, 1995, for a review). For these reasons, the goal of the current study was to develop, test, and present a larger pool of MBE stimuli in an effort to (1) demonstrate the generality of the phenomenon, and (2) expand the list of stimuli that produce the MBE.

\section{METHOD}

\section{Participants}

One hundred eighty students at the College of New Jersey 18 years or older participated in the experiment for partial course credit or extra credit.

\section{Materials}

The stimuli, consisting of blocking primes, positive primes, and corresponding word fragments, are displayed in the first three columns of Table 2. Positive primes were selected from the Nelson, McEvoy, and Schreiber (1998) University of South Florida fragment norm database. ${ }^{2}$ The rules described by Smith and Tindell (1997) were used to create the fragments. More specifically, two to four letters were deleted from positive primes, and blanks were inserted to form fragments. There were no more than two blanks in a row for any fragment. Therefore, the positive primes (e.g., BALCONY) always completed the word fragments (e.g., $B A L_{-} O N_{-}$).

Blocking primes are words that are orthographically similar to positive primes. These words were created by taking words from the Kučera and Francis (1967) corpus and matching them to positive primes. Blocking primes were selected based on the following criteria: (1) They did not complete the word fragments, (2) they were the same length as the fragment, (3) they had the same first letter as the positive prime, and (4) they shared at least three letters, with the shared letters in the same order for the prime words (Smith \& Tindell, 1997). For example, the blocking prime BALLOON does not complete its corresponding fragment $B A L_{-} O N_{-}$, is of the same length as the fragment, shares the first letter $(B)$, and shares four additional letters in the same order as the fragment $(A, L, O, N)$.

The 315 stimuli were randomly separated into nine lists of 105 stimuli sets containing a positive prime, blocking prime, and corresponding target fragment. Each list was broken up into thirds (35 words) to counterbalance the stimuli so that each set appeared as a positive prime, a blocking prime, and a control prime in equal numbers across participants in our sample.

\section{Procedure}

One of nine lists was randomly assigned to each participant. The experimental session consisted of a study phase, where participants studied 70 primes ( 35 blocking primes and 35 positive primes), and a test phase, where participants completed 105 word fragments.

The procedure for the study phase followed Smith and Tindell (1997). Stimuli were presented serially in the center of the computer screen in white uppercase letters on a black background. Participants were instructed to complete an affect rating task of a randomly intermixed list of 35 positive primes and 35 blocking primes. For the task, they had $5 \mathrm{sec}$ to indicate by keypress how the each word made them feel, with responses ranging from 1 (very bad) to 5 (very good). After five seconds elapsed without a judgment, the message "YOUR TIME IS UP" was displayed, and then the computer advanced to the next trial.

The procedure for the test phase followed Landau and Leynes (2006). One hundred and five fragments were presented serially in the center of the computer screen in white uppercase letters on a black background with an extra space in between each character to improve perception. Each character was the same size. An equal number of the test fragments (35) corresponded to the positive prime (that completed the fragment), blocking prime (orthographically similar to the fragment solution), and control conditions (solutions were unstudied). The instructions asked participants to type in missing letters in order to complete word fragments. The typed letters immediately replaced the blanks in the fragment. For the $B A L_{-}$ $O N_{-}$fragment example, typing " $\mathrm{C}$ " and then "Y" would display the completed word $B A L C O N Y$. Participants were instructed to avoid using slang words because they would be considered incorrect solutions. Typographical errors could be reduced by pressing the BACKSPACE key that cleared any entered letters and effectively redisplayed the original fragment. Participants were encouraged to be careful when entering letters because the computer automatically advanced to the next word when the last fragment blank was filled by keyboard input. After $10 \mathrm{sec}$ had elapsed without a complete solution, the message "YOUR TIME IS UP" was displayed, and the computer automatically advanced to the next fragment. Not allowing participants to retype the entire solution provided a very conservative estimate of the MBE magnitude because participants 


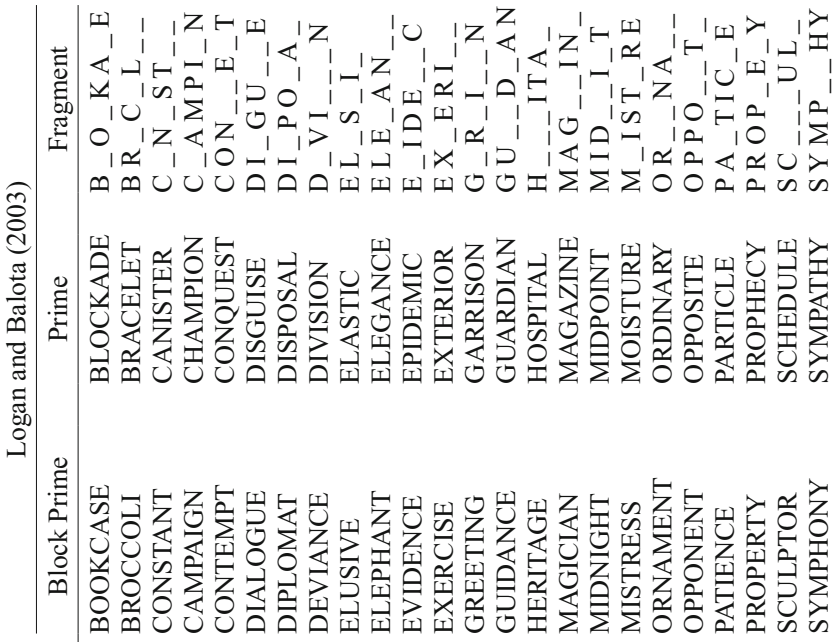

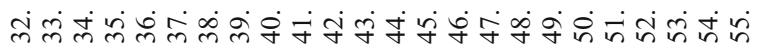

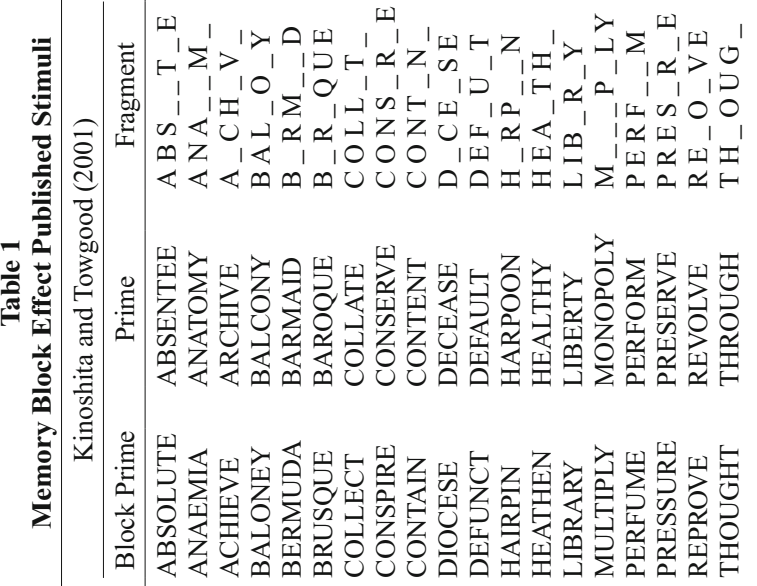

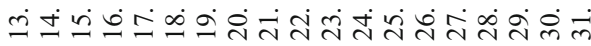

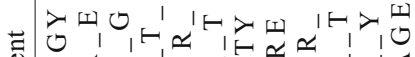

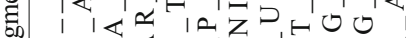

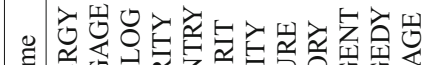

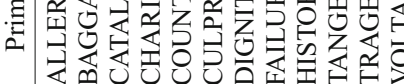

青

总

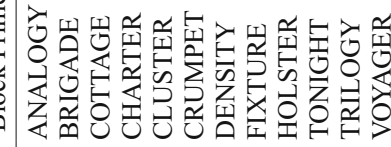

-4⿻一大 
could clearly see that a blocking prime cannot complete the fragment (Landau \& Leynes, 2006), whereas tests where solutions are retyped or copied might not lead to realization that the offered solution does not fit the fragment.

Fragment solutions were coded in the following manner: (1) correct response when the solution matched the positive prime or in some circumstances was a valid English word (e.g., from Kinoshita \& Towgood, 2001; the fragment $R E{ }_{-} O_{-} V E$ can be completed with RESOLVE or REVOLVE); (2) omission error when no letters were entered during the trial, (3) intrusion error when a nonword answer could be pronounced as a blocking word or included the same letters as the blocking word, and (4) other error when at least one blank was filled or a nonword was entered. Some examples of intrusion errors include (1) the solution $B A L \underline{O O N} \underline{N}$, because it is highly similar to the blocking prime (BALLOON) and very distinctive from any valid solution (BALCONY), and (2) the solution $A \underline{N B O R} \underline{M A L}$ because it is similar to the blocking prime (ABNORMAL) and not a valid solution (ARBOREAL).

\section{RESULTS}

The results are presented in two distinct sections in order to address the two purposes of this investigation. The first goal was to determine whether the MBE generalizes beyond select stimuli used in previous research (Kinoshita \& Towgood, 2001; Landau \& Leynes, 2006; Logan \& Balota, 2003; Lustig \& Hasher, 2001; Smith \& Tindell, 1997). To examine this question, the first set of results reports the effect of type of prime (blocking, control, positive) on average performance measures. These average performance measures were derived from averaging across individual stimuli for each participant and then averaging across participants to form the group means. Figure 1 displays this group averaged data in three panels: (A) the proportion of correctly completed fragments, (B) proportion of errors, and (C) response times (RTs).

The second goal of this study was to expand the pool of MBE stimuli. Accordingly, a second set of analyses examines mean fragment completion rates averaged across participants for each stimulus in an effort to evaluate the
MBE for each item in the corpus. These data are presented in the Appendix, which displays the proportion of accurate fragment completion in each of the three experimental conditions (blocking primes, control, and positive prime) for each of the individual MBE stimuli.

\section{The MBE in Average Performance Measures}

Correctly completed fragments. To test for the MBE, fragment completions were analyzed using a repeated measures ANOVA with one factor of prime (blocking, control, positive). The significant main effect of prime indicated that fragment completion varied across primes $\left[F(2,358)=661.35, p<.001, \eta^{2}=.79\right]$. Post hoc comparisons (Bonferroni-adjusted $F$ tests) revealed that exposure to blocking primes decreased the completion rate relative to the control condition $[F(1,179)=66.35, p<.001$, $\left.\eta^{2}=.27\right]$, whereas exposure to positive primes increased fragment completion relative to the control condition $\left[F(1,179)=713.32, p<.001, \eta^{2}=.80\right]$. Therefore, the basic memory block effects were replicated in this study.

Errors in fragment completion. Errors were analyzed using a 3 (prime: blocking, control, positive) $\times 3$ (error: omission, other, intrusion) repeated measures ANOVA. The significant main effect of prime $[F(2,358)=138.30$, $p<.001, \eta^{2}=.45$ ] indicated that errors varied as a function of prime, thereby mirroring analysis of completed fragments. ${ }^{3}$ Inspection of Figure 1 suggests that the significant error main effect $[F(2,358)=661.35, p<.001$, $\left.\eta^{2}=.79\right]$ resulted from fewer intrusions than the other types of errors. This result is not surprising given that the test format created a situation in which intrusion errors were obvious. Most importantly, a significant prime $\times$ error interaction $\left[F(4,716)=21.08, p<.001, \eta^{2}=.10\right]$ revealed that the pattern of errors varied for the primes. To explore the prime $\times$ error interaction, the effect of prime on each error was examined separately using Bonferronicorrected $F$ tests. These comparisons revealed that omissions $\left[F(2,358)=90.32, p<.001, \eta^{2}=.33\right]$, other errors
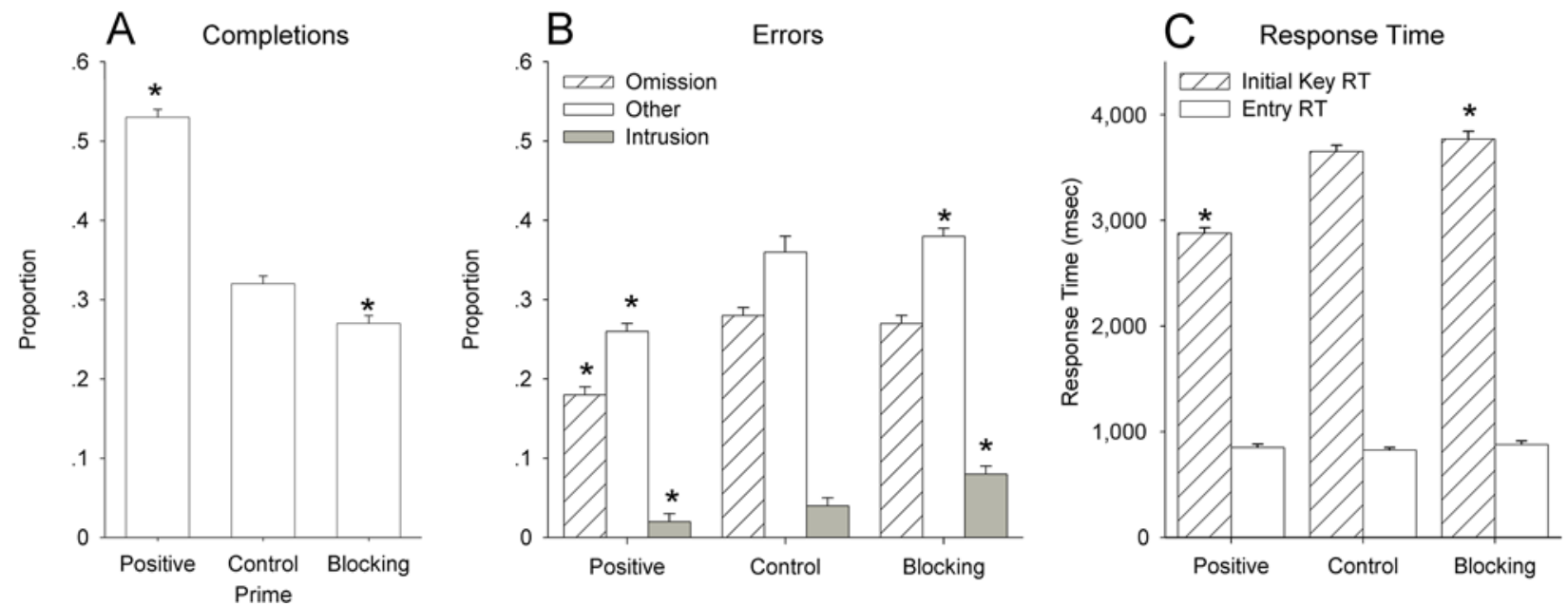

Figure 1. The proportions of accurately completed fragments (panel A), errors in fragment completion (panel B), and response times (panel C) as a function of the type of prime. Error bars are standard error of the mean. Asterisks in the figure indicate when a condition varied significantly from the control condition. 
$\left[F(2,358)=119.33, p<.001, \eta^{2}=.40\right]$, and intrusions $\left[F(2,358)=63.88, p<.001, \eta^{2}=.26\right]$ all varied as a function of prime. In order to understand errors across prime conditions completely, additional Bonferroni-corrected $F$ tests compared errors in the positive and blocking conditions to the control condition. The results of these contrasts revealed that positive primes reduced all three types of errors [omissions, $F(1,179)=130.54, p<.05, \eta^{2}=.42$; other, $F(1,179)=123.07, p<.05, \eta^{2}=.41$; intrusions, $\left.F(1,179)=11.81, p<.05, \eta^{2}=.06\right]$. Conversely, blocking primes increased intrusions $[F(1,179)=56.42, p<$ $\left..05, \eta^{2}=.24\right]$ and other errors $[F(1,179)=11.24, p<$ $\left..05, \eta^{2}=.06\right]$, but the number of omissions did not differ between the blocking and control conditions $[F(1,179)=$ $2.26, p=.13]$.

Collectively, these results are strong evidence that exposure to the blocking prime created a specific type of interference on fragment completion. Exposure to blocking primes did not lead people to simply withhold responses at a higher rate than in the control condition. Instead, people attempted to insert the blocking prime into the fragment, entered a few letters, or entered a nonword.

RTs for accurate solutions. RTs to make the initial keypress and the total time to enter an accurate solution were measured on each trial. The entry RT (time to enter a solution) was calculated by subtracting the initial keypress RT from the total RT. Trials on which no response was entered (i.e., omissions) were excluded from the RT analyses.

The analysis of initial keypress RT revealed a significant effect of prime $\left[F(2,358)=142.92, p<.001, \eta^{2}=.44\right]$. Post hoc comparisons indicated that positive primes resulted in faster RTs than the control condition $[F(1,179)=$ 245.92, $\left.p<.001, \eta^{2}=.58\right]$ and a marginally significant difference between the control and blocking conditions $\left[F(1,179)=3.58, p=.06, \eta^{2}=.02\right]$, which replicates the pattern observed by Logan and Balota (2003) using a different paradigm. There was no difference in entry RTs across the positive, control, and blocking conditions $[F(2,358)=$ $1.2, p=.30]$. Collectively, these results indicate that exposure to the blocking prime delayed the start to enter a solution, but the primes did not alter the time to enter a solution once participants began to solve the fragment.

\section{The MBE in Individual Stimuli}

The Appendix presents MBE stimuli, including positive primes, blocking primes, and target fragments, as well as the proportion of accurate fragment completion in each of the three conditions. The priming columns show the proportion of completion for positive and blocking conditions relative to the control condition that measured the base rate of completion. These values were calculated by subtracting the proportion of completions in the positive prime or the blocking prime conditions from the control condition. Inspection of these columns shows that some stimuli are more effective as blocking stimuli, others are more effective as positive primes, and a number are effective as both. In this study, blocking was defined as a negative value in the blocking column in the Appendix, which indicates that fewer of the fragments were completed when the blocking prime was studied relative to the control condition. The Appendix displays the stimuli tested sorted by the size of the block effect. One hundred eighty-five (59\%) of the stimuli tested produced blocking, and 130 stimuli did not produce blocking. A closer inspection of these stimuli revealed that 32 items did not produce blocking because the base completion rate in the control condition was 0 , and another 21 stimuli had a very low base completion rate (.05) making it difficult to observe blocking. These low rates indicate that for unknown reasons these fragments were difficult to complete for our participants. Excluding these stimuli reveals that $71 \%$ of the stimuli elicited blocking, whereas the remaining 30\% did not. This careful analysis quite clearly demonstrates that the MBE in a word fragment completion paradigm is very robust.

\section{DISCUSSION}

The purpose of the present investigation was twofold: (1) to determine the generality of the memory block effect, and (2) to expand the MBE stimuli. Using the rules originally described by Smith and Tindell (1997), a list of 315 sets of blocking primes, positive primes, and word fragments were created and tested.

Overall, several dependent measures indicated that the MBE does not depend on a specific set of stimuli. First, examination of completion rates revealed that exposure to the blocking primes decreased fragment completion rates relative to the control condition. Although the size of MBE in this study appears to be smaller than in other studies, it is important to emphasize that the test format and the unrefined stimulus pool provided a very conservative estimate of blocking. ${ }^{4}$ Second, examination of the errors revealed that omissions were equally likely in the blocking and control conditions, but intrusion errors were more likely when the blocking prime was studied. This is a profound result given that it was obvious to the participants that the blocking prime would not complete the fragment in the current study. Third, the analyses of the RTs recorded on trials when the correct solution was entered revealed that exposure to the blocking prime slowed the time to enter the first letter, but it did not affect total time to enter the solution. Collectively, these results are convincing evidence that exposure to orthographically similar primes slows retrieval when the fragment is solved and inhibits successful retrieval in many other cases because people tend to fixate on the blocking prime.

With regard to the second goal of the present study, blocking was observed for 185 new fragment sets. As a result, the entire list of MBE stimuli is sufficient for most basic memory studies as well as neuroimaging studies that use word fragment completions to measure blocking. This database is flexible because stimuli could be selected based on any number of criteria. For example, stimuli could be selected because the probability of blocking is greater than priming (or vice versa), the probabilities of priming and blocking are relatively equal, the base completion rates are equal, or to control for word frequency. 


\section{AUTHOR NOTE}

Correspondence concerning this article should be addressed to P. A. Leynes, Department of Psychology, College of New Jersey, P.O. Box 7718, Ewing, NJ 08628-0718 (e-mail: leynes@tcnj.edu).

\section{REFERENCES}

Kinoshita, S., \& Towgood, K. (2001). Effects of dividing attention on the memory-block effect. Journal of Experimental Psychology: Learning, Memory, \& Cognition, 27, 889-895.

KuČERa, H., \& Francis, W. N. (1967). Computational analysis of present-day American English. Providence, RI: Brown University Press.

LANDAU, J. D., \& Leynes, P. A. (2006). Do explicit memory manipulations affect the memory blocking effect? American Journal of Psychology, 19, 463-479.

Logan, J. M., \& Balota, D. A. (2003). Conscious and unconscious lexical retrieval blocking in younger and older adults. Psychology \& Aging, 18, 537-550.

Lustig, C., \& Hasher, C. (2001). Implicit memory is vulnerable to proactive interference. Psychological Science, 12, 408-412.

Nelson, D. L., McEvoy, C. L., \& Schreiber, T. A. (1998). The University of South Florida word association, rhyme, and fragment norms. Retrieved September 22, 2006, from w3.usf.edu/FreeAssociation

Pincton, T. W., Bentin, S., Berg, P., Donchin, E., Hillyard, S. A., Johnson, R., JR., ET AL. (2000). Guidelines for using human eventrelated potentials to study cognition: Recording standards and publication criteria. Psychophysiology, 37, 127-152.

RugG, M. D. (1995). ERP studies of memory. In M. D. Rugg \& M. G. H Coles (Eds.), Electrophysiology of mind: Event-related brain potentials and cognition. New York: Oxford University Press.

Smith, S. M., \& Tindell, D. R. (1997). Memory blocks in word fragment completion caused by involuntary retrieval of orthographically related primes. Journal of Experimental Psychology: Learning, Memory, \& Cognition, 23, 355-370.

\section{NOTES}

1. Kinoshita and Towgood (2001) used 30 stimuli sets - 19 of which were new and 10 that had been used by Smith and Tindell (1997). Logan and Balota (2003) used 36 stimuli sets -24 of which were new and 12 that had been used by Smith and Tindell. Lustig and Hasher (2001) increased the stimulus pool by using the Smith and Tindell stimuli in typical form and then reversing the positive primes and blocking primes to create an alternate list. For example, they used the blocking word "ANALOGY," the positive prime "ALLERGY," and the fragment "A L _ _ G Y" for one group of participants and the blocking word "ALLERGY," the positive prime "ANALOGY," and the fragment "A _ _ L

G Y" for another group. Landau and Leynes (2006) used the same 30 stimuli that had been used by Kinoshita and Towgood.

2. Conceptual primes that prime (at $\geq .3$ ) the positive primes in the list can be obtained by searching the Nelson, McEvoy, and Schreiber (1998) database.

3. Because the mean errors (overall) can be simply calculated as $1-$ $p$ (completion), we omit these means in order to conserve space.

4. Kinoshita and Towgood (2001) provide completion rates for the individual stimuli that they used, which can be used to estimate blocking effects for their stimuli. Inspection of their data also that suggests stimuli vary in their ability to elicit blocking; however, the percentage of noneffective stimuli in their article $(27 \%)$ appeared to be less than that observed in this study (41\%).

\section{ARCHIVED MATERIALS}

The following materials associated with this article may be accessed through the Psychonomic Society's Norms, Stimuli, and Data archive, www.psychonomic.org/archive.

To access these files, search the archive for this article using the journal name (Behavior Research Methods), the first author's name (Rass), and the publication year (2007).

FILE: Rass-BRM-2007.zip

DESCRIPTION: The compressed archive file contains two files:

Rass(2007).txt, containing memory block effect published stimuli

Rass(2007).xls, containing the above information in Excel spreadsheet format

AUTHOR's E-MAIL ADDRESS: leynes@tcnj.edu

(Manuscript received July 27, 2006; revision accepted for publication September 27, 2006.) 